\title{
Scalable Fabrication of Quasi-One-Dimensional Au Nanoribbons for Plasmonic Sensing
}

\author{
Chuanzhen Zhao ${ }^{1,2}$, Xiaobin Xu $\mathbf{X}^{1,2,3,4}$, Abdul Rahim Ferhan ${ }^{3}$, Naihao Chiang ${ }^{1,2,3}$, Joshua A.

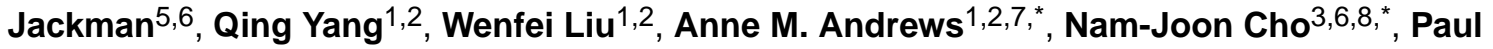 \\ S. Weiss ${ }^{1,2,9,10, *}$ \\ ${ }^{1}$ Department of Chemistry and Biochemistry, University of California, Los Angeles, Los Angeles, \\ California 90095, United States \\ ${ }^{2}$ California NanoSystems Institute, University of California, Los Angeles, Los Angeles, California \\ 90095, United States \\ ${ }^{3}$ School of Materials Science and Engineering, Nanyang Technological University, Singapore, \\ 639798, Singapore \\ ${ }^{4}$ Shanghai Key Lab. of D\&A for Metal-Functional Materials, School of Materials Science \& \\ Engineering, \& Institute for Advanced Study, Tongji University, Shanghai 201804, China \\ ${ }^{5}$ School of Chemical Engineering, Sungkyunkwan University, Suwon 16419, Republic of Korea \\ ${ }^{6}$ SKKU-UCLA-NTU Precision Biology Research Center, Sungkyunkwan University, Suwon 16419, \\ Republic of Korea
}

${ }^{7}$ Department of Psychiatry and Biobehavioral Sciences, Semel Institute for Neuroscience and Human Behavior, and Hatos Center for Neuropharmacology, University of California, Los Angeles, Los Angeles, California 90095, United States

${ }^{8}$ School of Chemical and Biomedical Engineering, Nanyang Technological University, 62 Nanyang Drive, 637459 Singapore

${ }^{9}$ Department of Bioengineering, University of California, Los Angeles, Los Angeles, California 90095, United States

${ }^{10}$ Department of Materials Science and Engineering, University of California, Los Angeles, Los Angeles, California 90095, United States

\section{Abstract}

\footnotetext{
*Corresponding Authors: aandrews@mednet.ucla.edu,njcho@ntu.edu.sg,psw@cnsi.ucla.edu. Author Contributions

The experiments were designed by CZ, XX, NJC, and PSW. Gold nanoribbon fabrication data were collected by CZ, XX, NC, QY, and WL, and were analyzed by all authors. Optical and plasmonic sensing measurements were performed and analyzed by ARF, JAJ, and NJC. Figures were prepared by CZ, XX, ARF. The manuscript was written by CZ, XX, ARF, JAJ, AMA, NJC, and PSW with assistance from all other authors.

Supporting Information

Materials and methods, scanning electron microscope image of Au nanoribbons after monolayer removal, energy-dispersive X-ray spectrum of an $\mathrm{In}_{2} \mathrm{O}_{3}$-coated Au nanoribbon substrate, flow-cell measurement set-up, extinction spectra of Au nanoribbons in buffer solutions with increasing glycerol concentrations (0-30 wt\%), time-resolved peak shifts during the adsorption of DOPC lipid vesicles or BSA.
} 
Plasmonic nanostructures have a wide range of applications including chemical and biological sensing. However, the development of techniques to fabricate sub-micron plasmonic structures over large scales remains challenging. We demonstrate a high-throughput, cost-effective approach to fabricate Au nanoribbons via chemical lift-off lithography (CLL). Commercial HD-DVDs were used as large-area templates for CLL. Transparent glass slides were coated with Au/Ti films and functionalized with self-assembled alkanethiolate monolayers. Monolayers were patterned with lines via CLL. The lifted-off, exposed regions of underlying Au were selectively etched into largearea grating-like patterns (200-nm linewidth; 400-nm pitch; 60-nm height). After removal of the remaining monolayers, a thin $\operatorname{In}_{2} \mathrm{O}_{3}$ layer was deposited and the resulting gratings were used as plasmonic sensors. Distinct features in the extinction spectra varied in their responses to refractive index changes in the solution environment with a maximum bulk sensitivity of $\sim 510 \mathrm{~nm} /$ refractive index unit. Sensitivity to local refractive index changes in the near-field was also achieved, as evidenced by real-time tracking of lipid vesicle or protein adsorption. These findings show how CLL provides a simple and economical means to pattern large-area plasmonic nanostructures for applications in optoelectronics and sensing.

\section{Graphical Abstract}

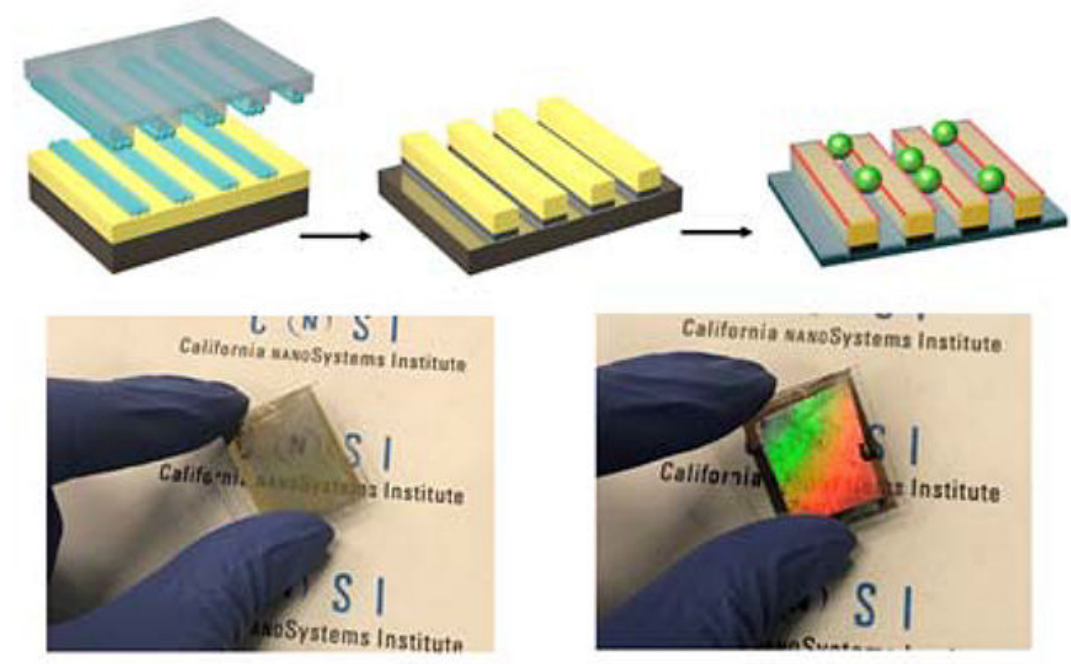

\section{Keywords}

Soft lithography; chemical lift-off lithography; nanoplasmonic; sensor; lipid vesicles

One of the most common measurement principles behind nanoplasmonic sensors is based on localized surface plasmon resonances (LSPRs), which result from interactions between light and noble metal nanostructures and lead to amplified electromagnetic fields in the vicinity of sensor surfaces. ${ }^{1,2}$ Typical analytes for nanoplasmonic sensors include ions, proteins, nucleic acids, viruses, exosomes, and liposomes. ${ }^{3-11}$ Depending on sensor design, nonspecific adsorption or specific analyte recognition via surface receptors can occur on sensor surfaces, leading to changes in the local refractive index within the amplified electromagnetic field near nanostructure surfaces. Such changes affect the plasmonic 
properties of the nanostructures and thereby give rise to changes in distinct features in the corresponding extinction spectra, including shifts in the LSPR resonance frequency (expressed as the peak shift wavelength). ${ }^{12}$ Nanoplasmonic sensors enable label-free and sensitive sensing capabilities for chemical and biological targets making these platforms attractive for applications related to food safety, defense, environmental protection, and biomedical devices. ${ }^{10,13-22}$

Fabrication of plasmonic substrates with nanometer-scale features is critical for practical applications. Producing sub-micron features often relies on state-of-the-art nanolithography tools, such as electron-beam or focused ion-beam lithographies. However, low throughput, high cost, and limited availability constrain scalable manufacturing of plasmonic nanostructures using the aforementioned methods. Soft lithographies, which utilize soft materials, e.g., polydimethylsiloxane (PDMS), to print or to replicate micro-/nanostructures, have emerged as alternatives to conventional photolithography for patterning at low cost and with high throughput. ${ }^{23-25}$ Nonetheless, it remains challenging to realize high-fidelity, submicron patterning using soft lithographies.

We have shown through chemical lift-off lithography (CLL) that we can produce large-area patterns having features with dimensions as small as tens of nanometers. ${ }^{26-31}$ The CLL process uses activated polymeric stamps to remove self-assembled monolayer (SAM) molecules from Au substrates selectively within (stamp-) contact areas. Sub-100 nm features are straightforwardly patterned using CLL, with features as small as $15 \mathrm{~nm}$ having been achieved. ${ }^{29,30,32}$ In addition, CLL can be used to pattern functional biomolecules for applications involving target recognition. ${ }^{28,29,33,34}$ Moreover, the chemical patterns produced by CLL serve as wet etching resists for transferring patterns to underlying materials, such as $\mathrm{Au}$ and $\mathrm{In}_{2} \mathrm{O}_{3} .{ }^{24,30}$

Herein, we report a scalable strategy for fabricating plasmonic nanostructures via CLL and the application of these substrates as nanoplasmonic sensors. Commercially available optical storage discs, which are inexpensive and ubiquitous, were used as nanostructured templates for making stamps for CLL. ${ }^{35}$ The CLL-patterned features functioned as etch resists to pattern underlying Au into plasmonic nanoribbons. Seminconductor-coated Au nanoribbons showed sensing capabilities with high sensitivity to refractive index changes relative to media composition or adsorption of biomolecules.

Optical storage media such as digital versatile discs (DVDs) and high-definition DVD (HDDVD) versions contain sub-micron periodic grating-like structures that can be used as templates for soft-lithography. As in our prior work, HD-DVDs were mechanically split into two layers to expose the layer of each disc having a nanotextured surface containing largearea concentric nanochannels. ${ }^{35}$ These nanostructured DVD layers were then used as masters for PDMS stamps. For high-quality stamp replication of the small features on DVDs, hard PDMS ( $h$-PDMS) was used as the stamp material.

The fabrication process for Au nanoribbon arrays using $h$-PDMS stamps templated from HD-DVD masters via CLL was similar to our previously reported procedure, ${ }^{35}$ with some modification. The current process is described in Figure 1 and the supplemental information. 
A DVD template is shown in Figure 2a. Atomic force microscopy (AFM) images of HDDVDs masters (Figure 2b,c) show large-area parallel nanoline features ( 200-nm linewidth and 400-nm periodicity). Hard PDMS stamps produced from the HD-DVD template are shown in Figure 2d. Corresponding AFM images of representative stamps confirm highquality feature replication (Figure 2e,f). After CLL, large-area SAM patterns were formed and characterized via scanning electron microscopy (SEM) (Figure 2g).

Selective Au etching produced sharp, uniform Au nanoribbon arrays that were continuous over large areas (tens of microns) (Figure 2h). The depth of etching was determined by measuring nanoribbon heights via AFM. (Figure 2i). Sputtering was used to coat $\operatorname{In}_{2} \mathrm{O}_{3}$ conformally on patterned Au nanoribbon surfaces. The Au nanoribbon patterns following monolayer removal (Figure S1) remained after $\mathrm{In}_{2} \mathrm{O}_{3}$ surface deposition (Figure 2j, 2k,2l, and Figure S2).

The $\operatorname{In}_{2} \mathrm{O}_{3}$-coated $\mathrm{Au}$ nanoribbon arrays were investigated for use as nanoplasmonic sensors. Apart from increasing platform stability, dielectric coatings, e.g., $\operatorname{In}_{2} \mathrm{O}_{3}$, enable indirect nanoplasmonic sensing and thus, characterization of interactions of biomolecules with a variety of materials beyond $\mathrm{Au} .{ }^{38,39}$ This approach has paved the way for studies involving biomolecule interactions with silicon and $\mathrm{TiO}_{2}$ surfaces (using other nanostructures as optical transducers). ${ }^{40-45}$ Here, arrays were fabricated on clear glass substrates to enable optical measurements in transmission mode. (The measurement setup is shown in Figure S3). Arrays on opaque substrates, e.g., Si, could be investigated using reflective mode.

Unlike flat substrates composed of thin Au films, which are typically used in surface plasmon resonance (SPR) sensors, nanostructured substrates lead to a unique interplay of plasmon modes involving localized and propagating surface plasmons. As such, nanoplasmonic sensors enable spectral features related to LSPR to be used to advantage in sensing applications. For example, nanoplasmonic sensors are more surface sensitive compared to conventional SPR sensors and are capable of tracking biomacromolecular interactions with high spatiotemporal resolution. ${ }^{15,36,37}$ In addition, measurements can be performed with a simple instrument setup consisting of a white light source and a spectrometer.

Although nanoplasmonic arrays on glass were translucent when viewed at an angle normal to the surface, they were reflective when tilted (Figure 3a). The latter is due to the high periodicity of the Au nanoribbons, which produces a diffraction grating. The UV-vis extinction spectra obtained in transmission mode exhibited five distinct features labelled peak 0, dip 1, peak 1, dip 2, and peak 2 at wavelengths of ca. 415, 503, 559, 581 and $649 \mathrm{~nm}$ in air, respectively (Figure 3b). With the exception of peak 0 , which is due to blue light absorption by $\mathrm{Au}$ and is non-plasmonic, the other peaks and dips underwent varying degrees of wavelength shift upon exposure to buffer solution due to the change in refractive index $v S$. air, ranging from ca. $30 \mathrm{~nm}$ (dip 1) to ca. $1 \mathrm{~nm}$ (peak 2) (Figure S4).

By considering the Au nanoribbons as analogous to high-aspect-ratio Au nanorods, peaks 1 and 2 were attributed to the transverse and longitudinal LSPR modes, respectively. ${ }^{46-48}$ However, contrary to discreet Au nanorod structures, where larger spectral shifts are 
typically observed at longer wavelengths, ${ }^{49,50}$ peak 2 and dip 2 , i.e., spectral features at longer wavelengths of the Au nanoribbon arrays, were less sensitive to bulk refractive-index changes compared to peak 1 and dip 1, i.e., spectral features at shorter wavelengths, as shown in Figure 3b. This result may be due to nanoribbons having ultra-high aspect ratios, i.e., the length of each ribbon was much larger than its width. Large-aspect-ratio geometries greatly diminish spectral contributions from the longitudinal LSPR mode. ${ }^{51-53}$

To characterize the sensitivity of the nanoribbon arrays to bulk refractive index (RI), we quantified extinction spectra feature shifts associated with different glycerol-water mixtures (0-30 wt\% glycerol), each of which has a well-defined refractive index.${ }^{54}$ From the evolution of the spectra, it was evident that dip 1 was the most sensitive feature, while peak 2 was the least sensitive to bulk RI changes (Figure 3c, full spectra in Figure S5). The bulk RI sensitivities for dip 1 and peak 2 were determined to be ca. $510 \mathrm{~nm} / \mathrm{RIU}$ (refractive index unit) and $60 \mathrm{~nm} / \mathrm{RIU}$, respectively (Figure 3d). The bulk RI sensitivity of dip 1 was significantly greater than other reported Au nanostructure arrays ${ }^{5,41,55-57}$ suggesting that our Au nanoribbon arrays might be particularly sensitive in chemical and biological sensing applications.

We investigated the nanoplasmonic characteristics of lipid vesicle interactions with $\operatorname{In}_{2} \mathrm{O}_{3^{-}}$coated Au-nanoribbon arrays (Figure 4a). We first determined the spectral noise by calculating the standard deviations of dip 1 and peak 2 wavelength positions over $3 \mathrm{~min}$ for arrays immersed in blank buffer. ${ }^{19}$ The spectral noise for dip 1 and peak 2 were $4.2 \times 10^{-2}$ $\mathrm{nm}$ and $1.0 \times 10^{-1} \mathrm{~nm}$, respectively. Based on the corresponding bulk refractive index sensitivity of the features, the spectral noise values of these two spectral features translate to a minimum practical resolution of $8.2 \times 10^{-5}$ RIU and $1.7 \times 10^{-3}$ RIU, respectively. ${ }^{58}$ Of note, despite relatively high spectral noise, the practical resolution of dip 1 is comparable to that obtained from commercially available oxide-coated nanoplasmonic substrates used in previous work, highlighting the advantage of high bulk refractive index sensitivity. ${ }^{19,59}$

Since dip 1 was the most responsive spectral feature to environmental changes based on bulk RI measurements, we characterized lipid vesicle adsorption via wavelength shifts for dip 1 (Figure $4 \mathrm{~b})$. Baseline spectra were obtained in buffer $(10 \mathrm{mM}$ tris(hydroxymethyl)aminomethane (Tris), pH 7.5, with $150 \mathrm{mM} \mathrm{NaCl}$ ) (Figure 4c). Next, 1,2-dioleoyl-sn-glycero-3-phosphocholine (DOPC) lipid vesicles $(0.2 \mathrm{mg} / \mathrm{mL})$ were introduced in Tris buffer at a continuous flow rate of $100 \mu \mathrm{L} / \mathrm{min}$. The time-resolved wavelength shifts indicated that the peak position of dip 1 increased linearly for $\sim 10 \mathrm{~min}$ before reaching a plateau. The time-dependent signal did not change upon subsequent buffer rinsing.

This result suggests a slow accumulation of DOPC lipid vesicles on nanoribbon surfaces. The mean absolute wavelength shift obtained from three different substrates was $1.2 \pm 0.1$ $\mathrm{nm}$. This shift is near the range observed in our previous work involving the adsorption of lipid vesicles on titanium oxide-coated Au nanodisk arrays, i.e., peak shift of 2.5-3.0 nm. 9,42,45 However, the wavelength shift normalized to the bulk RI sensitivity of dip 1 was significantly lower considering the higher bulk RI sensitivity value, i.e., $510 \mathrm{~nm} / \mathrm{RIU}$, compared to the bulk RI sensitivity of the analogous spectral dip associated with $\mathrm{Au}$ 
nanodisk arrays. i.e., $140 \mathrm{~nm} / \mathrm{RIU}$. The mean absolute wavelength shift for peak 2 from the Au nanoribbon arrays revealed a final value of $2.0 \pm 0.2 \mathrm{~nm}$ (Figure S6a). The slope (i.e., from baseline to plateau) can provide a series of quantitative information (Figure S7), such as initial rate and the number of intermediate steps, in any, involved in the biomolecular interaction, as we reported previously. ${ }^{29,38,41}$ Plasmonic features showed different responses to lipid vesicle adsorption with respect to their bulk sensitivities, suggesting a mismatch between bulk and surface sensitivities of the dips and peaks of the Au nanoribbon arrays.

To investigate this mismatch further, we characterized adsorption of a protein biomolecule, BSA, which is widely employed in a variety of applications, often as a blocking agent to prevent nonspecific adsorption on sensor surfaces. ${ }^{60-63}$ Recent efforts have relied on nanoplasmonic sensing strategies to quantify the adsorption of serum albumin on various surfaces for understanding adsorptive processes, ${ }^{40,44,59}$ as well as for investigating protein corona formation. ${ }^{64-67}$

After obtaining baseline signals in Tris buffer, $100 \mu \mathrm{M}$ BSA in Tris buffer was introduced at a flow rate of $100 \mu \mathrm{L} / \mathrm{min}$ and adsorption was monitored as a function of time on three different substrates. The time-resolved wavelength shift in the position of dip 1 revealed a steady initial increase, which plateaued at $1.2 \pm 0.2 \mathrm{~nm}$ (Figure 4d). Upon switching the flow back to Tris buffer, a sharp spike was reproducibly observed across different substrates prior to stabilization of the wavelength shift of dip 1 at $0.7 \pm 0.1 \mathrm{~nm}$. The net decrease in wavelength shift from $\sim 1.2 \mathrm{~nm}$ to $\sim 0.7 \mathrm{~nm}$ was likely due to the removal of weakly bound BSA molecules by washing, resulting in a decrease in local refractive index near the nanoplasmonic transducer surfaces.

The sharp, transient spike in the LSPR signal from $\sim 1.2 \mathrm{~nm}$ to $\sim 4.0 \mathrm{~nm}$ suggested a brief increase in local refractive index upon washing. As no additional protein was introduced into the system during this rinse step, the transient positive wavelength shift may have resulted from a redistribution of protein mass closer to the surfaces of the nanoplasmonic sensors into a region of higher electric field enhancement. In other words, the BSA molecules that remained strongly bound to the surface might have undergone post-adsorption redistribution, specifically protein spreading, wherein strongly adsorbed BSA molecules shifted nearer to the surface, on average. ${ }^{68}$ Weakly bound protein molecules were displaced leading to eventual net decreases in wavelength shifts.

The final wavelength shift of the dip 1 position is in good agreement with absolute values observed in our previous work, whereby the adsorption of bovine or human serum albumin onto $\mathrm{Si}$ or $\mathrm{TiO}_{2}$ surfaces led to LSPR peak shifts in the range $0.50-1.5 \mathrm{~nm} .{ }^{44,59}$ However, similar to DOPC lipid vesicle adsorption, the shift in the position of dip 1 resulting from BSA adsorption was relatively low when normalized to the bulk RI sensitivity. To determine whether this smaller shift was due to lower amounts of BSA adsorbed on indium oxide Au nanoribbon arrays or to differences in surface sensitivity, we extracted the time-resolved wavelength shifts for peak 2 during BSA adsorption (Figure S6b). The absolute peak 2 shift saturated close to $1.0 \mathrm{~nm}$, which is rather high considering the bulk RI sensitivity of this peak is only $\sim 60 \mathrm{~nm} / \mathrm{RIU}$, ruling out sparse BSA adsorption. 
We further investigated the dependence of the signal responses on DOPC lipid vesicle and BSA protein concentrations. The DOPC lipid vesicle concentration dependency curve extracted from dip 1 revealed a limit of detection (LOD) of $8.7 \mu \mathrm{g} / \mathrm{mL}$ and a dynamic range (DR) of $0.01-0.05 \mathrm{mg} / \mathrm{mL}$ (Figure 4d). In comparison, the LOD and DR for BSA protein adsorption based on the same spectral feature were $24 \mathrm{nM}(1.58 \mu \mathrm{g} / \mathrm{mL})$ and $0.1-100 \mathrm{uM}$ (6.6-6.6 $\times 10^{4} \mu \mathrm{g} / \mathrm{mL}$ ), respectively (Figure $4 \mathrm{f}$ ). On the other hand, the LOD and DR based on peak 2 for DOPC lipid vesicles were $16 \mu \mathrm{g} / \mathrm{mL}$ and $0.02-0.2 \mathrm{mg} / \mathrm{mL}$ (Figure S6c), and for BSA protein were $540 \mathrm{nM}(1.58 \mu \mathrm{g} / \mathrm{mL})$ and $0.1-100 \mu \mathrm{M}\left(6.6-6.6 \times 10^{4} \mu \mathrm{g} / \mathrm{mL}\right)$, respectively (Figure S6d). Overall, dip 1 represents a more sensitive spectral feature than peak 2 for quantification of DOPC lipid vesicle and BSA protein adsorption at lower concentrations. However, peak 2 offers better quantification than dip 1 for resolving DOPC lipid vesicle concentration differences over a wider range, while the opposite is true for BSA protein. This distinction likely arises from different plasmon modes and probing volumes attributed to each spectral feature and highlights the merits of using this type of Au nanoribbon array.

The variations in the final shifts in the positions of dip $1 \mathrm{vs}$. peak 2 during DOPC vesicle and BSA adsorption, which did not scale proportionally with their respective bulk RI sensitivities confirm a mismatch between surface and bulk sensitivities of the dip 1 and peak 2 spectral features (Table S1). Thus, while dip 1 exhibited higher bulk RI sensitivity than peak 2, surface sensitivities were lower for dip 1. This difference might arise from an enhanced electric field in the dip-sensitive region that extends over larger sensing volumes compared to the electric field of the peak-sensitive region, which is focused at the $\mathrm{Au}-$ solution interface and covers smaller sensing volumes. For nanoplasmonic sensors, the correlation between bulk and surface sensitivities is complex, as there are several interacting factors of importance, including nanoplasmonic transducer geometry, orientation, and dielectric coating. ${ }^{69,70}$

Particularly in the context of sensing applications, it is important to distinguish between bulk and surface sensitivities since changes in local refractive indices close to sensor surfaces are specific to molecular adsorption events. A more pronounced spike was observed in the shift of the position of dip 1 (Figure 4d) compared to the peak 2 shift (Figure S6b) during buffer rinsing after BSA adsorption. This result suggests that the post-adsorption protein spreading on the $\mathrm{In}_{2} \mathrm{O}_{3}$-coated Au nanoribbon surfaces is significant and extends beyond the sensing volume of the peak-sensitive region. By contrast, the overall profiles of the shifts in the positions of the principal dips and peaks for DOPC vesicle adsorption indicated no significant lipid redistribution after rinsing.

Taken together, we have demonstrated the capability of Au nanoribbon arrays with a thin indium oxide coating prepared via a simple fabrication approach to detect the adsorption of biomolecules in real time. These arrays enabled post-adsorption changes in protein distribution to be monitored, providing new information on the interactions between serum albumin and oxide surfaces. While the importance of distinguishing bulk and surface sensitivities has been discussed, ${ }^{71}$ our work highlights mismatches in the respective sensitivities of two plasmonic spectral features from the same sensor array, which were used to compare qualitatively the extent of adsorption of two different biomolecules. 
In summary, we report a high-throughput, large-scale, and low-cost nanofabrication approach to produce Au plasmonic sensors. Commercially available HD-DVDs were employed as large-area templates for soft lithography. Stamps patterned using HD-DVD templates possessed large-area nanoline features (200-nm linewidths with 400-nm pitch) and were used to fabricate subwavelength Au plasmonic sensors.

Optical characterization of indium oxide-coated Au nanoarrays revealed plasmon-active spectral features with varying bulk refractive index sensitivities $(\sim 60-500 \mathrm{~nm} / \mathrm{RIU})$. We utilized Au nanoribbon arrays for real-time sensing of DOPC vesicle or BSA adsorption, and exploited the mismatch in bulk and surface sensitivities between key dip- and peaksensitive regions to distinguish adsorptive properties of these two types of biomolecules. Together, these results demonstrate that scalable patterning by chemical lift-off lithography provides a straightforward approach for large-area plasmonic nanostructure fabrication with applications in optoelectronics and biointerfacial science. In this proof-of-concept work, we studied the interactions between biomolecules and nonfunctionalized nanostructure surfaces. Selective sensing could be achieved on functionalized nanoplasmonic surfaces, e.g., with antibodies or DNA, towards antigen or complementary DNA detection, respectively. 10,58,72-74 Further work using the platform will use target-specific biomolecules, including DNA and antigens, to build a universal biosensor platform for broad applications.

\section{Supplementary Material}

Refer to Web version on PubMed Central for supplementary material.

\section{ACKNOWLEDGMENTS}

This work was supported by the United States National Science Foundation (CMMI-1636136) and National Institute on Drug Abuse (DA045550), and the SKKU-UCLA-NTU Precision Biology Research Center. We acknowledge the use of the facilities and thank the staff of the Nanoelectronics Research Facility, Electron Imaging Center, Nano \& Pico Characterization Lab, and Integrated Systems Nanofabrication Cleanroom of the California

NanoSystems Institute. The authors thank Dr. Jeffrey J. Schwartz for useful discussions and assistance.

\section{REFERENCES}

(1). Anker JN; Hall WP; Lyandres O; Shah NC; Zhao J; Van Duyne RP Nat. Mater. 2008, 7, 442-453. [PubMed: 18497851]

(2). Dahlin AB; Wittenberg NJ; Hook F; Oh SH Nanophotonics 2013, 2, 83-101. [PubMed: 24159429]

(3). Sharpe JC; Mitchell JS; Lin L; Sedoglavich N; Blaikie RJ Anal. Chem. 2008, 80, 2244-2249. [PubMed: 18288819]

(4). Yang JC; Ji J; Hogle JM; Larson DN Nano Lett. 2008, 8, 2718-2724. [PubMed: 18710296]

(5). Lee S-W; Lee K-S; Ahn J; Lee J-J; Kim M-G; Shin Y-B ACS Nano 2011, 5, 897-904. [PubMed: 21222487]

(6). Zheng YB; Payton JL; Chung CH; Liu R; Cheunkar S; Pathem BK; Yang Y; Jensen L; Weiss PS Nano Lett. 2011, 11, 3447-3452. [PubMed: 21749070]

(7). Kumar K; Dahlin AB; Sannomiya T; Kaufmann S; Isa L; Reimhult E Nano Lett. 2013, 13, 61226129. [PubMed: 24188470]

(8). Valsecchi C; Brolo AG Langmuir 2013, 29, 5638-5649. [PubMed: 23488664]

(9). Jackman JA; Yorulmaz Avsar S; Ferhan AR; Li D; Park JH; Zhdanov VP; Cho N-J Anal. Chem. 2017, 89, 1102-1109. [PubMed: 27983791] 
(10). Oliverio M; Perotto S; Messina GC; Lovato L; De Angelis F ACS Appl. Mater. Inter. 2017, 9 , 29394-29411.

(11). Cetin AE; Iyidogan P; Hayashi Y; Wallen M; Vijayan K; Tu E; Nguyen M; Oliphant A ACS Sensors 2018, 3, 561-568. [PubMed: 29488377]

(12). Willets KA; Van Duyne RP Annu. Rev. Phys. Chem. 2007, 58, 267-297. [PubMed: 17067281]

(13). Anker JN; Hall WP; Lyandres O; Shah NC; Zhao J; Van Duyne RP Nat. Mater. 2008, 7, 442-453. [PubMed: 18497851]

(14). Spackova B; Wrobel P; Bockova M; Homola J Proc. IEEE 2016, 104, 2380-2408.

(15). Jackman JA; Rahim Ferhan A; Cho N-J Chem. Soc. Rev. 2017, 46, 3615-3660. [PubMed: 28383083]

(16). Escobedo C; Brolo AG; Gordon R; Sinton D Nano Lett. 2012, 12, 1592-1596. [PubMed: 22352888]

(17). Tokel O; Inci F; Demirci U Chem. Rev. 2014, 114, 5728-5752. [PubMed: 24745365]

(18). Jeong JW; Yang SR; Hur YH; Kim SW; Baek KM; Yim S; Jang HI; Park JH; Lee SY; Park CO; Jung YS Nat. Commun. 2014, 5, 5387. [PubMed: 25382491]

(19). Wang X; Chang TW; Lin G; Gartia MR; Liu GL Anal. Chem. 2017, 89, 611-615. [PubMed: 27976865]

(20). Ballard ZS; Shir D; Bhardwaj A; Bazargan S; Sathianathan S; Ozcan A ACS Nano 2017, 11, 2266-2274. [PubMed: 28128933]

(21). Belushkin A; Yesilkoy F; Altug H ACS Nano 2018, 12, 4453-4461. [PubMed: 29715005]

(22). Narasimhan V; Siddique RH; Lee JO; Kumar S; Ndjamen B; Du J; Hong N; Sretavan D; Choo H Nat. Nanotechnol. 2018, 13, 512-519. [PubMed: 29713074]

(23). Qin D; Xia Y; Whitesides GM Nat. Protocols 2010, 5, 491-502. [PubMed: 20203666]

(24). Saavedra HM; Mullen TJ; Zhang P; Dewey DC; Claridge SA; Weiss PS Rep. Prog. Phys. 2010, 73, 036501.

(25). Shuster MJ; Vaish A; Cao HH; Guttentag AI; McManigle JE; Gibb AL; Martinez-Rivera M; Nezarati RM; Hinds JM; Liao WS; Weiss PS; Andrews AM Chem. Comm. 2011, 47, 1064110643. [PubMed: 21874174]

(26). Liao WS; Cheunkar S; Cao HH; Bednar HR; Weiss PS; Andrews AM Science 2012, 337, $1517-$ 1521. [PubMed: 22997333]

(27). Kim J; Rim YS; Chen H; Cao HH; Nakatsuka N; Hinton HL; Zhao C; Andrews AM; Yang Y; Weiss PS ACS Nano 2015, 9, 4572-4582. [PubMed: 25798751]

(28). Abendroth JM; Nakatsuka N; Ye M; Kim D; Fullerton EE; Andrews AM; Weiss PS ACS Nano 2017, 11, 7516-7526. [PubMed: 28672111]

(29). Xu X; Yang Q; Cheung KM; Zhao C; Wattanatorn N; Belling JN; Abendroth JM; Slaughter LS; Mirkin CA; Andrews AM; Weiss PS Nano Lett. 2017, 17, 3302-3311. [PubMed: 28409640]

(30). Zhao C; Xu X; Yang Q; Man T; Jonas SJ; Schwartz JJ; Andrews AM; Weiss PS Nano Lett. 2017, 17, 5035-5042. [PubMed: 28737930]

(31). Cheung KM; Stemer DM; Zhao C; Young TD; Belling JN; Andrews AM; Weiss PS ACS Mater. Lett. 2019, 2, 76-83.

(32). Andrews AM; Liao WS; Weiss PS Acct. Chem. Res. 2016, 49, 1449-1457.

(33). Cao HH; Nakatsuka N; Liao W-S; Serino AC; Cheunkar S; Yang H; Weiss PS; Andrews AM Chem. Mater. 2017, 29, 6829-6839.

(34). Cao HH; Nakatsuka N; Serino AC; Liao WS; Cheunkar S; Yang H; Weiss PS; Andrews AM ACS Nano 2015, 9, 11439-11454. [PubMed: 26426585]

(35). Zhao C; Xu X; Bae SH; Yang Q; Liu W; Belling JN; Cheung KM; Rim YS; Yang Y; Andrews AM; Weiss PS Nano Lett. 2018, 18, 5590-5595. [PubMed: 30060654]

(36). Junesch J; Emilsson G; Xiong K; Kumar S; Sannomiya T; Pace H; Voros J; Oh SH; Bally M; Dahlin AB Nanoscale 2015, 7, 15080-15085. [PubMed: 26351000]

(37). Ferhan AR; Jackman JA; Malekian B; Xiong K; Emilsson G; Park S; Dahlin AB; Cho NJ Anal. Chem. 2018, 90, 7458-7466. [PubMed: 29806449] 
(38). Langhammer C; Larsson EM; Kasemo B; Zoric I Nano Lett. 2010, 10, 3529-3538. [PubMed: 20718400]

(39). Li JQ; Ye J; Chen C; Li Y; Verellen N; Moshchalkov VV; Lagae L; Van Dorpe P ACS Photonics 2015, 2, 425-431.

(40). Zen F; Karanikolas VD; Behan JA; Andersson J; Ciapetti G; Bradley AL; Colavita PE Langmuir 2017, 33, 4198-4206. [PubMed: 28398737]

(41). Zan GH; Jackman JA; Kim SO; Cho NJ Small 2014, 10, 4828-4832. [PubMed: 25079046]

(42). Jackman JA; Zhdanov VP; Cho N-J Langmuir 2014, 30, 9494-9503. [PubMed: 25035920]

(43). Jackman JA; Spackova B; Linardy E; Kim MC; Yoon BK; Homola J; Cho N-J Chem. Comm. 2016, 52, 76-79. [PubMed: 26466086]

(44). Jackman JA; Ferhan AR; Yoon BK; Park JH; Zhdanov VP; Cho NJ Anal. Chem. 2017, 89, 12976-12983. [PubMed: 29111680]

(45). Ferhan AR; Jackman JA; Cho N-J Phys. Chem. Chem. Phys. 2017, 19, 2131-2139. [PubMed: 28045148]

(46). Wu H-Y; Chu H-C; Kuo T-J; Kuo C-L; Huang MH Chem. Mater. 2005, 17, 6447-6451.

(47). Yasukuni R; Ouhenia-Ouadahi K; Boubekeur-Lecaque L; Félidj N; Maurel F; Métivier R; Nakatani K; Aubard J; Grand J Langmuir 2013, 29, 12633-12637. [PubMed: 24070218]

(48). Wang Y-N; Wei W-T; Yang C-W; Huang MH Langmuir 2013, 29, 10491-10497. [PubMed: 23924308]

(49). Miller MM; Lazarides AA J. Phys. Chem. B 2005, 109, 21556-21565. [PubMed: 16853799]

(50). Lee K-S; El-Sayed MA J. Phys. Chem. B 2006, 110, 19220-19225. [PubMed: 17004772]

(51). Link S; El-Sayed MA J. Phys. Chem. B 1999, 103, 8410-8426.

(52). Schmucker AL; Harris N; Banholzer MJ; Blaber MG; Osberg KD; Schatz GC; Mirkin CA ACS Nano 2010, 4, 5453-5463. [PubMed: 20738131]

(53). Zhang S; Chen L; Huang Y; Xu H Nanoscale 2013, 5, 6985-6991. [PubMed: 23800794]

(54). Lide DR CRC Press, Boca Raton, USA 2001, 76, 1995-1996.

(55). Xiong K; Emilsson G; Dahlin AB Analyst 2016, 141, 3803-3810. [PubMed: 26867475]

(56). Ode K; Honjo M; Takashima Y; Tsuruoka T; Akamatsu K ACS Appl. Mater. Inter. 2016, 8, 20522-20526.

(57). Malekian B; Xiong K; Emilsson G; Andersson J; Fager C; Olsson E; Larsson-Langhammer E; Dahlin A Sensors 2017, 17, 1444.

(58). Homola J Chem. Rev. 2008, 108, 462-493. [PubMed: 18229953]

(59). Ferhan AR; Jackman JA; Sut TN; Cho NJ Sensors (Basel) 2018, 18, 1283.

(60). Sweryda-Krawiec B; Devaraj H; Jacob G; Hickman JJ Langmuir 2004, 20, 2054-2056. [PubMed: 15835649]

(61). Reimhult K; Petersson K; Krozer A Langmuir 2008, 24, 8695-8700. [PubMed: 18646724]

(62). Jeyachandran YL; Mielczarski JA; Mielczarski E; Rai BJ Colloid Interface Sci. 2010, 341, 136142.

(63). Park JH; Sut TN; Jackman JA; Ferhan AR; Yoon BK; Cho N-J Phys. Chem. Chem. Phys. 2017, 19, 8854-8865. [PubMed: 28294278]

(64). Frost R; Wadell C; Hellman A; Molander S; Svedhem S; Persson M; Langhammer C ACS Sensors 2016, 1, 798-806.

(65). Frost R; Langhammer C; Cedervall T Nanoscale 2017, 9, 3620-3628. [PubMed: 28247891]

(66). Ke PC; Lin S; Parak WJ; Davis TP; Caruso F ACS Nano 2017, 11, 11773-11776. [PubMed: 29206030]

(67). Xu M; Soliman MG; Sun X; Pelaz B; Feliu N; Parak WJ; Liu SJ ACS Nano 2018, 12, $10104-$ 10113. [PubMed: 30212621]

(68). Park JH; Jackman JA; Ferhan AR; Ma GJ; Yoon BK; Cho NJ ACS Appl. Mater. Inter. 2018, 10, 32047-32057.

(69). Li J; Ye J; Chen C; Li Y; Verellen N; Moshchalkov VV; Lagae L; Van Dorpe P ACS Photonics 2015, 2, 425-431.

(70). Li J; Chen C; Lagae L; Van Dorpe PJ Phys. Chem. C 2015, 119, 29116-29122. 
(71). Szunerits S; Boukherroub R Chem. Comm. 2012, 48, 8999-9010. [PubMed: 22806135]

(72). Belushkin A; Yesilkoy F; Gonzalez-Lopez JJ; Ruiz-Rodriguez JC; Ferrer R; Fabrega A; Altug H Small 2019, e1906108. [PubMed: 31830370]

(73). Špačková B; Lynn NS; Slabý J; Šípová H; Homola J ACS Photonics 2018, 5, 1019-1025.

(74). Yoo SY; Kim DK; Park TJ; Kim EK; Tamiya E; Lee SY Anal. Chem. 2010, 82, 1349-1357. [PubMed: 20092310] 


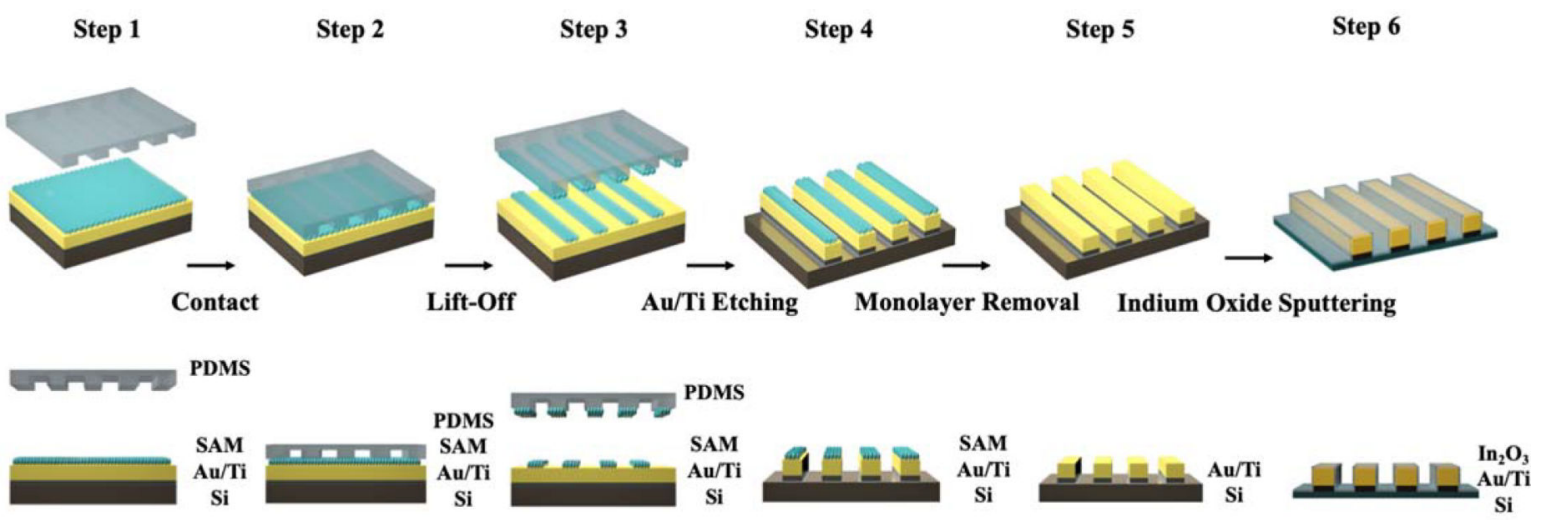

Figure 1.

Schematic of Au nanoscale grating fabrication. Step 1: The Au (50 nM) and Ti (10 nm) layers deposited using electron-beam evaporation onto glass substrates were functionalized with self-assembled monolayers (SAMs) of 11-mercapto-1-undecanol. Step 2: Patterned hard polydimethylsiloxane ( $h$-PDMS) stamps activated by oxygen plasma were brought into conformal contact with functionalized substrates. Step 3: Molecules in the contacted areas were removed from each surface to form nanoribbon patterns. Step 4: Selective etching of the exposed Au regions. Step 5: Remaining SAM molecules were removed to obtain bare Au nanoscale gratings. Step 6: A layer of $\mathrm{In}_{2} \mathrm{O}_{3}(10 \mathrm{~nm})$ was sputtered to cover the sensor surface. 

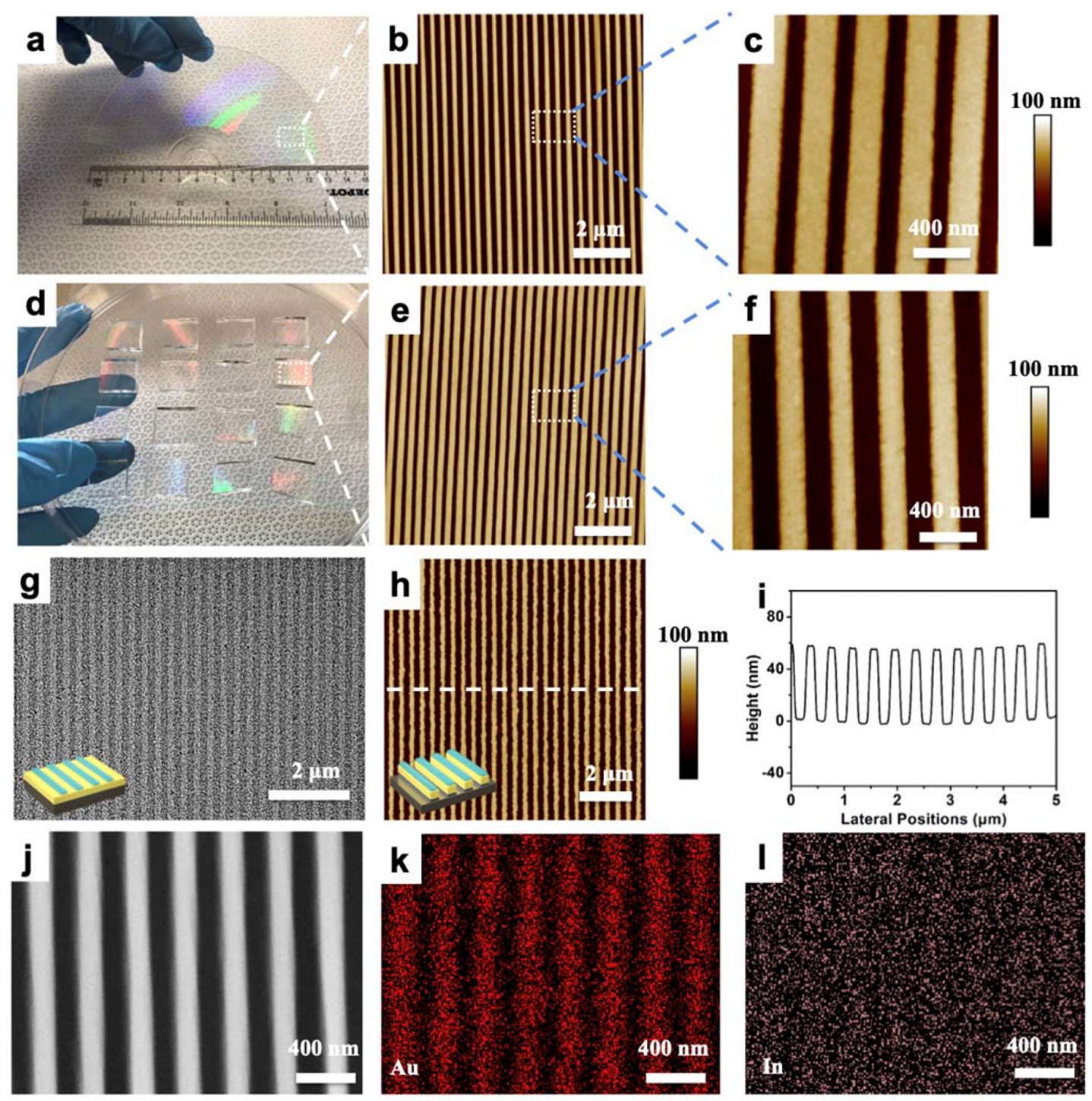

Figure 2.

(a) Photograph of a HD-DVD master after separation from a commercial disk. (b, c) Atomic force microscope images of HD-DVD master. (d) Photograph of hard polydimethylsiloxane ( $h$-PDMS) stamps prepared using a single HD-DVD master, each measuring approximately $1.5 \mathrm{~cm} \times 1.5 \mathrm{~cm}$. (e, f) Atomic force microscope images of patterned $h$-PDMS. (g) A scanning electron microscope (SEM) image of a self-assembled-monolayer nanoribbon pattern, where the darker lines represent regions where molecules were removed to expose the underlying Au surface. (h) Atomic force microscope image of Au nanoribbons with 200$\mathrm{nm}$ widths and a 400-nm pitch. (i) Profile of Au nanoribbons indicating heights of $\sim 60 \mathrm{~nm}$. (j) An SEM image of Au nanoribbons after $\mathrm{In}_{2} \mathrm{O}_{3}$ deposition. (k, l) Energy-dispersive X-ray mapping of $\mathrm{Au}$ and $\mathrm{In}$, respectively, after conformal sputtering of $\mathrm{In}_{2} \mathrm{O}_{3}$ at the same spot shown in $\mathrm{j}$. 
a

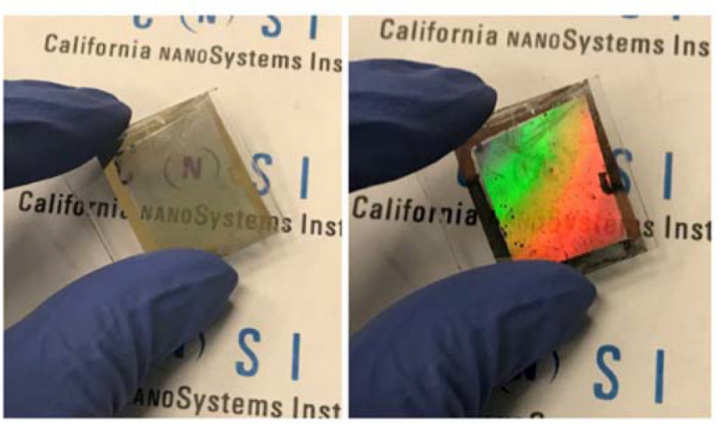

C

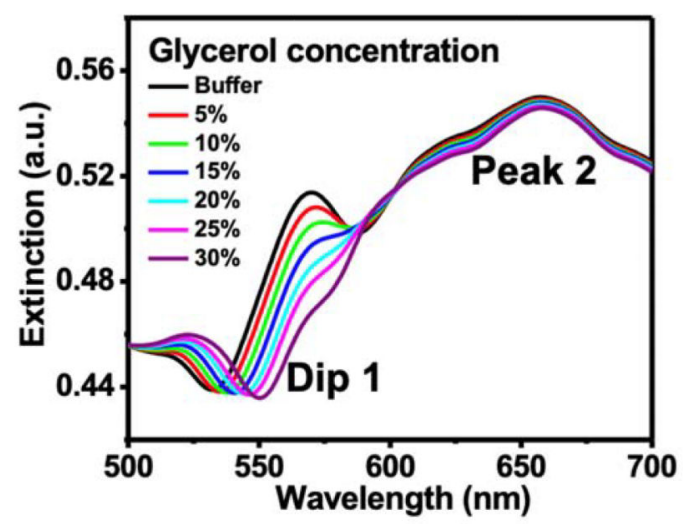

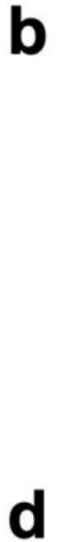
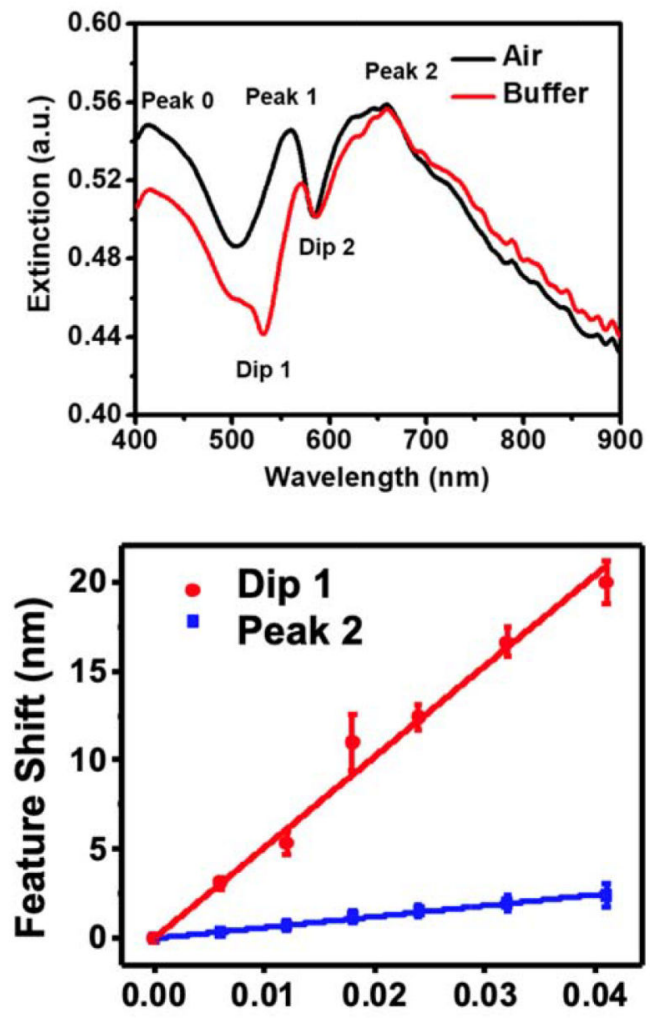

Change in Bulk Refractive Index (RIU)

Figure 3.

(a) Photograph of Au nanoribbons fabricated on glass slides $(\sim 3 \mathrm{~cm} \times 3 \mathrm{~cm})$ viewed from different perspectives showing transparency $v s$. reflectivity depending on the viewing angle.

(b) Extinction spectra of a representative Au nanoribbon array in air (black trace) vs. buffer (10 mM tris(hydroxymethyl)aminomethane, $\mathrm{pH} 7.5$, with $150 \mathrm{mM} \mathrm{NaCl}$ ) (red trace). (c) Representative extinction spectra of a Au nanoribbon array exposed to buffer solutions with increasing glycerol concentrations (0-30 wt\%) during bulk refractive index sensitivity characterization. (d) Bulk refractive index sensitivities of the dip 1 and peak 2 features from spectra obtained at each of the glycerol concentrations. Sensitivities were determined from the slopes of the curves. Data are from $N=3$ substrates produced from different fabrication runs. Error bars are standard errors of the means and too small to be visualized in some cases. 
a

Step 1
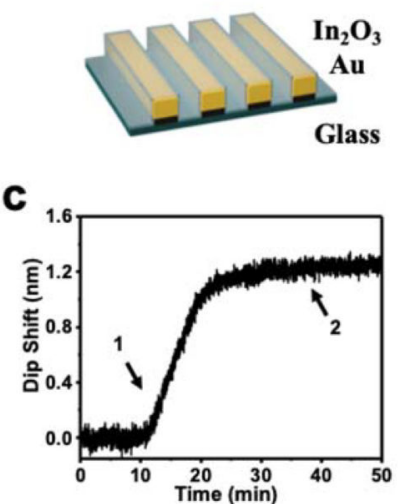

Step 2

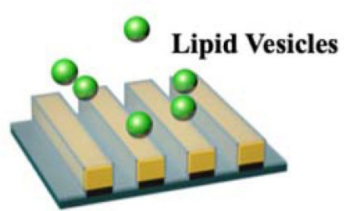

d

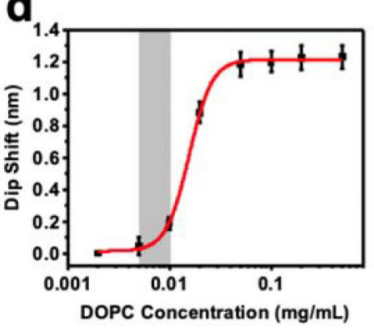

Step 3

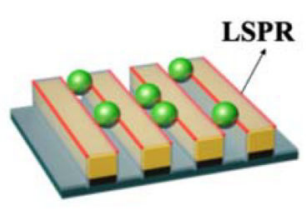

e

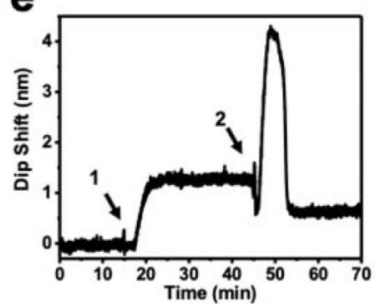

b

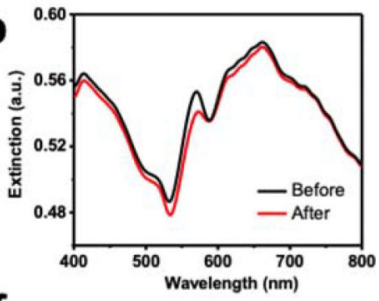

f

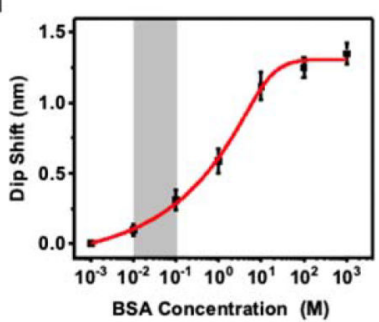

Figure 4.

(a) Schematic illustration of lipid vesicle detection using a Au plasmonic sensor. A solution containing 1,2-dioleoyl-sn-glycero-3-phosphocholine (DOPC) vesicles was continuously flowed across a nanoribbon array (step 2). Some liposomes adsorbed noncovalently to the oxide surface, where red lines indicate the localized surface plasmon resonance (step 3). (b) Extinction spectra showing spectral shifts before and after vesicle adsorption. Time-resolved shifts in the positions of dip 1 during the adsorption of (c) DOPC lipid vesicles or (e) bovine serum albumin (BSA). Arrows indicate the time points where (1) flow was switched from Tris buffer to buffer containing DOPC lipid vesicles or BSA and (2) flow was switched back to buffer. Concentration dependence of the net wavelength shift (before washing) upon addition of (d) DOPC lipid vesicles or (f) BSA protein. The respective limits of detection (LOD) in grey are included and represent the lowest detectable concentration that would produce a wavelength shift corresponding to $3 \sigma$, where $\sigma$ is the standard deviation of the background signal in the presence of blank buffer. Data are from $N=3$ runs on the same substrate and error bars represent standard deviations. 